\title{
Estimated evapotranspiration of rice based on pan evaporation as a surrogate to lysimeter measurement
}

\begin{abstract}
Reference crop evapotranspiration (ET o), used to determine actual crop evapotranspiration, is often estimated from pan evaporation (EP) data. However, uncertainties in the relationship between ET o and EP often result in unreliable estimate of crop evapotranspiration. This study investigated the relationship between measured and estimated crop evapotranspirations, ET $\mathrm{m}$ and ET e, respectively, at tillering (9ї 30 days after transplanting, DAT) and midgrowth (51ï 72 DAT) stages of a rice variety. ET m was measured with a Marriott Tube-type Micro-lysimeter (hereafter referred to Micro-lysimeter) in a ponded rice field and ET e was estimated from EP, which was measured by employing the US Weather Bureau Class óAô Evaporation Pan (hereafter referred to Class A Evaporation Pan). A strong linear relation (r 2 $=0.89)$ at the tillering stage and a weak relation $(\mathrm{r} 2=0.48)$ at the mid-growth stage were

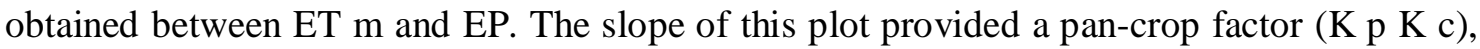
which was 0.81 at the tillering stage and 0.79 at the mid-growth stage. The ET e versus ET $\mathrm{m}$ relationship was also strongly linear $(\mathrm{r} 2=0.90)$ at the tillering stage but weakly linear ( $2=$ $0.50)$ at the mid-growth stage. The pan-based method thus provided reliable estimates of evapotranspiration during the tillering stage of rice.
\end{abstract}

Keyword: Class 'A' Pan evaporation; Evapotranspiration; Growth stage; Micro-lysimeter; Rice 